\title{
On the Performance of Successive Interference Cancellation in D2D-enabled Cellular Networks
}

\author{
Chuan $\mathrm{Ma}^{1}$, Weijie $\mathrm{Wu}^{1}$, Ying $\mathrm{Cui}^{1}$, Xinbing Wang ${ }^{1,2}$ \\ 1. School of Electronic Info. \& Electrical Eng., Shanghai Jiao Tong University, China \\ 2. National Mobile Communications Research Laboratory, Southeast University, China \\ Email: \{oknewkimi, weijiewu, cuiying, xwang8\}@sjtu.edu.cn
}

\begin{abstract}
Device-to-device (D2D) communication underlaying cellular networks is a promising technology to improve network resource utilization. In D2D-enabled cellular networks, the interference among spectrum-sharing links is more severer than that in traditional cellular networks, which motivates the adoption of interference cancellation techniques such as successive interference cancellation (SIC) at the receivers. However, to date, how SIC can affect the performance of D2D-enabled cellular networks is still unknown. In this paper, we present an analytical framework for studying the performance of SIC in large-scale D2D-enabled cellular networks using the tools from stochastic geometry. To facilitate the interference analysis, we propose the approach of stochastic equivalence of the interference, which converts the two-tier interference (interference from both the cellular tier and D2D tier) to an equivalent single-tier interference. Based on the proposed stochastic equivalence models, we derive the general expressions for the successful transmission probabilities of cellular uplinks and D2D links with infinite and finite SIC capabilities respectively. We demonstrate how SIC affects the performance of large-scale D2D-enabled cellular networks by both analytical and numerical results.
\end{abstract}

\section{INTRODUCTION}

Recently, there has been a rapid increase in the demand of local area services and proximity services (ProSe) among the highly-capable user equipments (UEs) in cellular networks. In this context, a new technology called device-to-device (D2D) communication, which enables direct communication between UEs that are in proximity, has been proposed and has strongly appealed to both academia [1], [2] and industry [3], [4]. The integration of D2D communication to cellular networks holds the promise of many types of advantages [2]: allowing for high-rate low-delay low-power transmission for proximity services, increasing frequency reuse factor and network capacity, facilitating new types of peer-to-peer services, etc.

However, the introduction of D2D communication also brings a number of technical challenges, such as device discovery, mode selection and interference management. Interference management is a major issue in D2D-enabled cellular networks, since D2D links share the same spectrum resource with regular cellular links and the interference among the spectrum-sharing links severely hampers the performance of the network. To guarantee reliable communications in D2Denabled cellular networks, extensive research has been undertaken on the topic of interference management. Most proposed schemes can be classified into three categories: (1) Interference avoidance: orthogonal time-frequency resource allocation schemes are adopted to avoid interference between D2D and cellular links [5]; (2) Interference coordination: intelligent power control and link scheduling schemes are employed to mitigate the interference between D2D and cellular links [6], [7]; and (3) Interference cancellation: advanced signal processing techniques are applied at cellular and/or D2D links to cancel the interfering signals [8], [9]. In this paper, we focus on the topic of interference cancellation in D2D-enabled cellular networks.

Interference cancellation (IC) is regarded as a promising technique to reduce interference and improve network capacity. Using interference cancellation techniques, the interfering signals can be regenerated and subsequently canceled from the desired signal [10]. Best known interference cancellation techniques include successive interference cancellation (SIC), parallel interference cancellation (PIC) and iterative interference cancellation (IIC) [11]. The key advantage of SIC compared to other interference cancellation techniques is that the SIC receiver is architecturally similar to traditional non-SIC receivers in terms of hardware complexity and cost [11], as it uses the same decoder to decode the composite signal at different stages and neither complicated decoders nor multiple antennas are required. Furthermore, it is known that SIC can achieve the Shannon capacity region boundaries for both the broadcast and multiple access networks. As such, SIC has been widely studied and recently implemented in commercial wireless systems such as IEEE 802.15.4. However, to date, most analytical results on SIC are for ad hoc and cellular networks. It is still unknown how SIC can improve the performance of large-scale D2D-enabled cellular networks, in which the interference among spectrum-sharing links is more severer than that in traditional ad hoc and cellular networks. In this paper, we present an analytical framework to evaluate how SIC affects the performance of large-scale D2D-enabled cellular networks. The main contributions of this paper are summarized as follows.

(1) We model a large-scale D2D-enabled cellular network without SIC capabilities via stochastic geometry, and derive the general expressions for the successful transmission probabilities of cellular uplinks and D2D links. Furthermore, to simplify the interference analysis in the network, we propose the approach of stochastic equivalence of the interference. By this approach, the two-tier interference (interference from the cellular tier and D2D tier) can be represented by an 
equivalent single-tier interference that maintains the same stochastic characteristics as the two-tier interference.

(2) Based on the stochastic equivalence models, we derive the general expressions for the successful transmission probabilities of cellular uplinks and D2D links with infinite and finite SIC capabilities respectively. We demonstrate the effect of SIC on the performance of large-scale D2D-enabled cellular networks by analytical and numerical results.

The rest of this paper is organized as follows. Section II presents the related work. Section III describes the system model. Sections IV and V analyze the successful transmission probabilities in D2D-enabled cellular networks without and with SIC respectively and present the numerical results. Section VI concludes the paper. A summary of the notations used in this paper is given in Table I.

\section{RELATED WORK}

D2D communication. There have been numerous studies on interference management for D2D communications. $\mathrm{Xu}$ et al. [5] proposed a combinatorial auction approach to allocate orthogonal resources between cellular and D2D users. Kaufman et al. [6] presented an opportunistic communication scheme in which the D2D network can communicate as a fully loaded cellular network. Pei and Liang [7] designed a spectrum sharing protocol that enables D2D users to communicate bi-directionally while assisting the two-way communications between the base station and cellular users. Min et al. [8] designed an interference cancellation scheme that exploits a retransmission of the interference from the base station. Ma et al. [9] proposed two superposition coding-based cooperative relaying schemes to exploit the transmission opportunities for D2D users without deteriorating the performance of cellular users.

Successive interference cancellation. Very recently, there is a growing interest to exploit SIC at the physical layer to improve network performances at upper layers. In [12], Gelal et al. proposed a topology control framework for exploiting the benefits of multi-packet reception using SIC in multiuser MIMO networks. In [13], Jiang et al. combines SIC and interference avoidance to improve the throughput of a multihop network. In [14], Xu et al. developed a decentralized power allocation scheme to achieve the maximum throughput for random access systems with SIC receivers. In [15], Lv et al. proposed two layered models to characterize the impact of SIC, and presented corresponding link scheduling schemes under these models. In [16], Mollanoori and Ghaderi studied the uplink scheduling problems for networks supporting SIC, and derived the optimal decoding order of the concurrent transmissions.

Stochastic geometry for modeling wireless networks. As a mathematical tool to study random spatial patterns, stochastic geometry can be used to model and analyze the interference, connectivity and coverage in large-scale wireless networks [17]. Most of the literature in the area of modeling networks via stochastic geometry focus on ad hoc [18], [19] and cellular [20], [21] networks. Recently, stochastic geometry has
Table I: Notations used in the paper

\begin{tabular}{|c|c|}
\hline Notation & Description \\
\hline \hline$\Phi_{c}$ & Poisson point process of cellular users (density $\lambda_{c}$ ) \\
\hline$\Phi_{d}$ & Poisson point process of D2D transmitters (density $\left.\lambda_{d}\right)$ \\
\hline$\Phi_{c-i n t f}^{\mathrm{eq}}$ & $\begin{array}{c}\text { Equivalent Poisson point process of } \\
\left.\text { the interferers for cellular links (density } \lambda_{c-i n t f}^{\mathrm{eq}}\right)\end{array}$ \\
\hline$\Phi_{d-i n t f}^{\mathrm{eq}}$ & $\begin{array}{c}\text { Equivalent Poisson point process of } \\
\left.\text { the interference for D2D links (density } \lambda_{d-i n t f}^{\text {eq }}\right)\end{array}$ \\
\hline$P_{c}$ & Transmission power of cellular users \\
\hline$P_{d}$ & Transmission power of D2D users \\
\hline$\alpha$ & Path loss exponent $\left(\delta=\frac{2}{\alpha}\right)$ \\
\hline$T$ & SIR threshold for successful transmission \\
\hline$p_{c}$ & Successful transmission prob. of cellular links without SIC \\
\hline$p_{d}$ & Successful transmission prob. of D2D links without SIC \\
\hline$p_{c}^{\text {SIC }}, p_{c}^{N-\text { SIC }}$ & $\begin{array}{c}\text { Successful transmission prob. of cellular links } \\
\text { with infinite and (finite) } N \text {-level SIC }\end{array}$ \\
\hline$p_{d}^{\text {SIC }}, p_{d}^{M-\text { SIC }}$ & $\begin{array}{c}\text { Successful transmission prob. of D2D links } \\
\text { with infinite and (finite) } M \text {-level SIC }\end{array}$ \\
\hline
\end{tabular}

also been employed to model D2D-enabled cellular networks [22]-[24]. In [22]-[24], the cellular and D2D networks were modeled by independent PPPs, and their SINR distributions were derived without considering interference cancellation techniques. The stochastic geometry-based analysis of SIC has been presented in literature [25]-[29]. In [25], [26], simplified SIC models were given by assuming that the interference greater than a threshold can be completely canceled. Exact SIC models were investigated for ad hoc network in [27], [28] and for cellular networks in [29]. Different from [27]-[29], in this paper, we focus on the analysis of the effect of SIC for D2Denabled cellular networks. Since the stochastic characteristics of heterogeneous networks (comprising ad hoc users and cellular users) are quite different from those of homogeneous networks (comprising only ad hoc users or cellular users), the analysis of SIC in this paper is more challenging than that in [27]-[29].

\section{System MODEL}

In this section, we elaborate on the network model and describe the SIC scheme.

\section{A. Network Model}

We consider a spectrum-sharing D2D-enabled cellular network consisting of both cellular users and D2D users over a large two-dimensional space, and focus on the uplink transmission for cellular users. The cellular users are assumed to be spatially distributed as a homogeneous Poisson point process (PPP) $\Phi_{c}$ with density $\lambda_{c}$, and an independent collection of base stations (BSs) is assumed to be located according to some independent stationary point process $\Phi_{b}$. We assume that each cellular user is associated with its nearest base station, and each base station has only one active uplink cellular user scheduled. Under such assumptions, each base station can be considered to be uniformly distributed in the Voronoi cell of its associated cellular user, as shown in Fig.1. It is noted that the orthogonal scheduling policy leads to coupling between the locations of cellular users and those of base stations. Nevertheless, it has been shown that the 


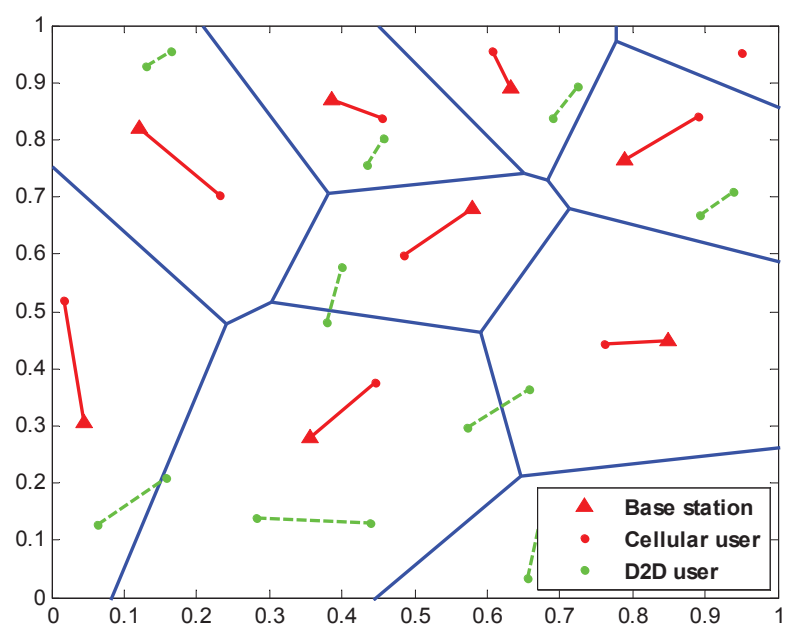

Figure 1: Network model.

dependence introduced by coupling has negligible effects on the performance analysis [21], [29], Therefore, for analytical tractability, we assume that the point processes of cellular users and base stations are independent.

The D2D transmitters in the network are assumed to be distributed according to a homogeneous PPP $\Phi_{d}$ with density $\lambda_{d}$. For a given D2D transmitter, its associated receiver is assumed to be located at a distance $l$ away with isotropic direction, where $l$ is Rayleigh distributed:

$$
f_{l}(l)=2 \pi \lambda_{d} l e^{-\pi \lambda_{d} l^{2}} .
$$

This Rayleigh distribution assumption is of practical interest and is employed in many works [22]-[24]. Other distributions of $l$ can be easily incorporated into the framework.

The transmission powers are assumed to be $P_{c}$ at uplink cellular users and $P_{d}$ at D2D transmitters respectively. We adopt a unified channel model that comprises standard path loss and Rayleigh fading for both cellular and D2D links: given transmission power $P$ of the transmitter located at $x_{i}$, the received power at the receiver located at $x_{j}$ can be expressed as $P h\left\|x_{i}-x_{j}\right\|^{-\alpha}$, where $h$ is the fading factor following an exponential distribution with unit mean, i.e. $h \sim \exp (1)$, and $\alpha>2$ is the path loss exponent. In later parts of this paper we use $\delta$ to denote $\frac{2}{\alpha}$ for brevity of expressions. In addition, as interference dominates noise in most modern cellular networks, we consider the network to be interferencelimited.

\section{B. SIC Technique}

SIC is a promising interference cancellation technique that has been widely studied for wireless networks. The basic concept of SIC is to regenerate the interfering signals and subsequently cancel them from the received composite signal, so as to improve the signal-to-interference ratio (SIR) of the desired signal. In this technique, the SIC receiver first decodes the strongest interfering signal by treating other signals as noise. Then it regenerates the analog signal from the decoded signal, and cancels it from the received composite signal. After

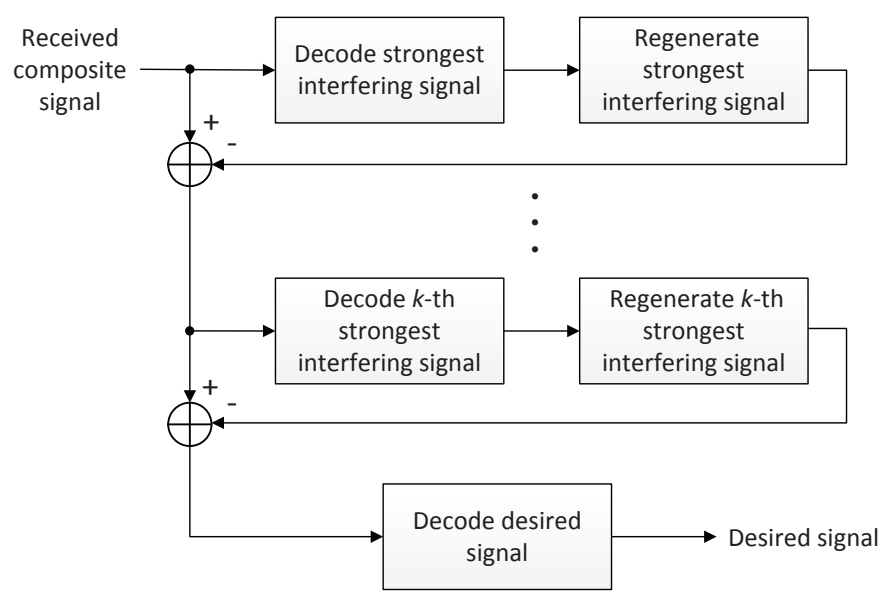

Figure 2: Schematic diagram of SIC process.

this stage, the remaining signal is free from the interference of the strongest interfering signal. Then, the SIC receiver proceeds to decode, regenerate and cancel the second strongest interfering signaling from the remaining signal and so forth, until the desired signal can be decoded. The schematic diagram of SIC process is shown in Fig.2.

In this paper, we assume that the SIC technique is employed at both cellular receivers (BSs) and D2D receivers. For cellular receivers, we consider the cases of infinite and finite SIC capabilities respectively, and study the successful transmission probabilities of cellular links for each case. However, for D2D receivers, due to their limited computational capabilities, we focus only on the case of finite SIC capability.

\section{Network Performance Without SiC}

In this section, we consider the scenario that neither the cellular receivers (BSs) nor the D2D receivers have SIC capabilities, and derive the successful transmission probabilities of both cellular and D2D links. We also analyze the stochastic equivalence of interference in the network, which is essential for the SIC analysis in later sections.

\section{A. Successful Transmission Probability of Cellular Links}

Without loss of generality, we conduct the analysis on a typical cellular link that comprises a typical BS located at the origin and its associated cellular user located at a random distance $r$ away. Under the nearest-BS association policy, the random variable $r$ can be shown to be Rayleigh distributed and its probability density function (pdf) follows [21]:

$$
f_{r}(r)=2 \pi \lambda_{c} r e^{-\pi \lambda_{c} r^{2}} .
$$

Denote the fading factor of the typical cellular link by $g_{0}$, which is i.i.d exponential with $g_{0} \sim \exp (1)$. Then, the received SIR of the typical cellular link, i.e., the received SIR at the typical BS, can be expressed as

$$
\mathrm{SIR}_{c}=\frac{P_{c} g_{0} r^{-\alpha}}{I_{c}},
$$


where

$$
I_{c}=\sum_{x_{i} \in \Phi_{c} \backslash\left\{x_{0}\right\}} P_{c} g_{i}\left\|x_{i}\right\|^{-\alpha}+\sum_{y_{i} \in \Phi_{d}} P_{d} h_{i}\left\|y_{i}\right\|^{-\alpha}
$$

is the cumulative interference from all other cellular users (except the typical cellular user $x_{0}$ ) that are located at $x_{i}$ with fading factor $g_{i}$ and D2D transmitters that are located at $y_{i}$ with fading factor $h_{i}{ }^{1}$.

The successful transmission probability of cellular links can be defined as

$$
p_{c} \triangleq \mathbb{P}\left[\mathrm{SIR}_{c}>T\right],
$$

where $T$ is the SIR threshold. The expression of $p_{c}$ is given by the following theorem.

Theorem 1. The successful transmission probability of cellular links without SIC capability is

$$
p_{c}=\frac{\lambda_{c}}{\lambda_{c}(\mu+1)+\lambda_{d}\left(\frac{P_{d}}{P_{c}}\right)^{\delta} \nu},
$$

where

$$
\begin{aligned}
\mu & =\frac{\delta}{1-\delta} T \cdot{ }_{2} F_{1}(1,1-\delta ; 2-\delta ;-T), \\
\nu & =T^{\delta} \Gamma(1-\delta) \Gamma(1+\delta),
\end{aligned}
$$

and ${ }_{2} F_{1}(\cdot), \Gamma(\cdot)$ are respectively the Hypergeometric function and Gamma function.

Proof: Starting from the definitions of $p_{c}$, we have

$$
\begin{aligned}
p_{c} & \triangleq \mathbb{P}\left[\operatorname{SIR}_{c}>T\right] \\
& =\mathbb{E}_{r, I_{c}}\left[\mathbb{P}_{g_{0}}\left[\operatorname{SIR}_{c}>T\right]\right] \\
& =\mathbb{E}_{r, I_{c}}\left[\mathbb{P}_{g_{0}}\left[g_{0}>P_{c}^{-1} \operatorname{Tr}^{\alpha} I_{c}\right]\right] \\
& \stackrel{(a)}{=} \mathbb{E}_{r, I_{c}}\left[\exp \left(-P_{c}^{-1} \operatorname{Tr}^{\alpha} I_{c}\right)\right] \\
& \stackrel{(b)}{=} \mathbb{E}_{r}\left[\mathcal{L}_{I_{c}}\left(P_{c}^{-1} \operatorname{Tr}^{\alpha}\right)\right] \\
& =\int_{0}^{\infty} \mathcal{L}_{I_{c}}\left(P_{c}^{-1} \operatorname{Tr}^{\alpha}\right) \cdot f_{r}(r) \mathrm{d} r .
\end{aligned}
$$

(a) follows from the Rayleigh distribution assumption of channel fading. In $(b), \mathcal{L}_{I_{c}}(\cdot)$ denotes the Laplace transform of $I_{c}$. Let $I_{c}=I_{c-c}+I_{c-d}$, where $I_{c-c}=$ $\sum_{x_{i} \in \Phi_{c} \backslash\left\{x_{0}\right\}} P_{c} g_{i}\left\|x_{i}\right\|^{-\alpha}$ and $I_{c-d}=\sum_{y_{i} \in \Phi_{d}} P_{d} h_{i}\left\|y_{i}\right\|^{-\alpha}$ denote the interference from cellular links and D2D links respectively. Then it is straightforward to get

$$
\mathcal{L}_{I_{c}}(s)=\mathcal{L}_{I_{c-c}}(s) \cdot \mathcal{L}_{I_{c-d}}(s) .
$$

The Laplace transform of $I_{c-c}$ is given by

$$
\mathcal{L}_{I_{c-c}}(s)=\mathbb{E}\left[\exp \left(-s \sum_{x_{i} \in \Phi_{c} \backslash\left\{x_{0}\right\}} P_{c} g_{i}\left\|x_{i}\right\|^{-\alpha}\right)\right]
$$

\footnotetext{
${ }^{1}$ To distinguish different links, in this paper we use $g \sim \exp (1), h \sim$ $\exp (1)$ to represent the fading factors of links related to cellular transmitters (cellular users) and D2D transmitters respectively. It is noted that there is no essential distinction between these two symbols.
}

$$
\begin{aligned}
& =\mathbb{E}_{\Phi_{c}}\left[\prod_{x_{i} \in \Phi_{c} \backslash\left\{x_{0}\right\}} \mathbb{E}_{g}\left[\exp \left(-s P_{c} g_{i}\left\|x_{i}\right\|^{-\alpha}\right)\right]\right] \\
& \stackrel{(c)}{=} \exp \left(-\lambda_{c} \int_{\Phi_{c} \cap \bar{B}(o, r)}\left(1-\mathbb{E}_{g}\left[e^{-s P_{c} g_{i}\left\|x_{i}\right\|^{-\alpha}}\right]\right) \mathrm{d} x_{i}\right) \\
& =\exp \left(-\lambda_{c} \int_{\Phi_{c} \cap \bar{B}(o, r)}\left(1-\frac{1}{1+s P_{c}\left\|x_{i}\right\|^{-\alpha}}\right) \mathrm{d} x_{i}\right) \\
& \stackrel{(d)}{=} \exp \left(-\lambda_{c} \cdot 2 \pi \int_{r}^{\infty} \frac{v}{1+s^{-1} P_{c}^{-1} v^{\alpha}} \mathrm{d} v\right) \\
& \stackrel{(e)}{=} \exp \left(-\lambda_{c} \pi \frac{\delta}{1-\delta} s P_{c} r^{2-\alpha}{ }_{2} F_{1}\left(1,1-\delta ; 2-\delta ;-\frac{s P_{c}}{r^{\alpha}}\right)\right) .
\end{aligned}
$$

(c) follows from the probability generating functional (PGFL) of PPP [30]: $\mathbb{E}\left[\prod_{x \in \Phi} f(x)\right]=\exp \left(-\lambda \int_{\mathbb{R}^{2}}(1-f(x)) \mathrm{d} x\right)$. $(d)$ follows from the double integral in polar coordinates. $(e)$ follows from the definite integral [31, 3.194.2]: $\int_{b}^{\infty} \frac{v}{1+a v^{\alpha}} \mathrm{d} v=\frac{1}{\alpha} \cdot \frac{b^{2-\alpha}}{a\left(1-\frac{2}{\alpha}\right)}{ }_{2} F_{1}\left(1,1-\frac{2}{\alpha} ; 2-\frac{2}{\alpha} ;-\frac{1}{a b^{\alpha}}\right)$. Similarly, we have

$$
\begin{aligned}
& \mathcal{L}_{I_{c-d}}(s)=\mathbb{E}\left[\exp \left(-s \sum_{y_{i} \in \Phi_{d}} P_{d} h_{i}\left\|y_{i}\right\|^{-\alpha}\right)\right] \\
& =\exp \left(-\lambda_{d} \int_{\mathbb{R}^{2}}\left(1-\mathbb{E}_{h}\left[\exp \left(-s P_{d} h_{i}\left\|y_{i}\right\|^{-\alpha}\right)\right]\right) \mathrm{d} y_{i}\right) \\
& =\exp \left(-\lambda_{d} \int_{\mathbb{R}^{2}}\left(1-\frac{1}{1+s P_{d}\left\|y_{i}\right\|^{-\alpha}}\right) \mathrm{d} y_{i}\right) \\
& =\exp \left(-\lambda_{d} \cdot 2 \pi \int_{0}^{\infty} \frac{u}{1+s^{-1} P_{d}^{-1} u^{\alpha}} \mathrm{d} u\right) \\
& =\exp \left(-\lambda_{d} \pi\left(s P_{d}\right)^{\delta} \Gamma(1-\delta) \Gamma(1+\delta)\right)
\end{aligned}
$$

By plugging (11) (12) into (10) and letting $s=P_{c}^{-1} T r^{\alpha}$, we get

$$
\mathcal{L}_{I_{c}}\left(P_{c}^{-1} \operatorname{Tr}^{\alpha}\right)=\exp \left(-\pi\left[\lambda_{c} \mu+\lambda_{d}\left(\frac{P_{d}}{P_{c}}\right)^{\delta} \nu\right] r^{2}\right),
$$

where $\mu=\frac{\delta}{1-\delta} T \cdot{ }_{2} F_{1}(1,1-\delta ; 2-\delta ;-T), \nu=T^{\delta}$. $\Gamma(1-\delta) \Gamma(1+\delta)$. Then by plugging (2) (13) into (9), we complete the proof.

\section{B. Successful Transmission Probability of D2D Links}

We conduct the analysis on a typical D2D link that comprises a typical D2D transmitter located at some point in the network and a typical D2D receiver located at a random distance $l$ away. Shift the coordinates such that the typical D2D receiver is located at the origin ${ }^{2}$, and denote the fading factor of the typical D2D link by $h_{0}, h_{0} \sim \exp (1)$. Then, the received SIR of the typical D2D link can be expressed as

$$
\mathrm{SIR}_{d}=\frac{P_{d} h_{0} l^{-\alpha}}{I_{d}}
$$

\footnotetext{
${ }^{2}$ It is noted that the translations do not change the distribution of PPP [32]
} 
where

$$
I_{d}=\sum_{y_{i} \in \Phi_{d} \backslash\left\{y_{0}\right\}} P_{d} h_{i}\left\|y_{i}\right\|^{-\alpha}+\sum_{x_{i} \in \Phi_{c}} P_{c} g_{i}\left\|x_{i}\right\|^{-\alpha}
$$

is the cumulative interference from all other D2D transmitters (except the typical D2D transmitter located at $y_{0}$ ) that are located at $y_{i}$ with fading factor $h_{i}$ and cellular users that are located at $x_{i}$ with fading factor $g_{i}$.

The successful transmission probability of D2D links can be defined as

$$
p_{d} \triangleq \mathbb{P}\left[\mathrm{SIR}_{d}>T\right],
$$

where $T$ is the SIR threshold. Note that the same SIR threshold $T$ is assumed for cellular and D2D links. The expression of $p_{d}$ is given by the following theorem.

Theorem 2. The successful transmission probability of $D 2 D$ links without SIC capability is

$$
p_{d}=\frac{\lambda_{d}}{\lambda_{d}(\nu+1)+\lambda_{c}\left(\frac{P_{c}}{P_{d}}\right)^{\delta} \nu},
$$

where $\nu$ is given in (8).

Proof: Starting from the definitions of $p_{d}$ and $\mathrm{SIR}_{d}$,

$$
\begin{aligned}
p_{d} & \triangleq \mathbb{P}\left[\operatorname{SIR}_{d}>T\right] \\
& =\mathbb{E}_{l, I_{d}}\left[\mathbb{P}_{h_{0}}\left[\mathrm{SIR}_{d}>T\right]\right] \\
& =\mathbb{E}_{l}\left[\mathcal{L}_{I_{d}}\left(P_{d}^{-1} T l^{\alpha}\right)\right] \\
& =\int_{0}^{\infty} \mathcal{L}_{I_{d}}\left(P_{d}^{-1} T l^{\alpha}\right) \cdot f_{l}(l) \mathrm{d} l .
\end{aligned}
$$

Following approaches similar to those in previous proofs and Slivnyak's theorem [30]: $\mathbb{P}^{! x}=\mathbb{P}$, we have

$$
\begin{aligned}
& \mathcal{L}_{I_{d}}(s)=\mathbb{E}_{I_{d}}\left[\exp \left(-s I_{d}\right)\right] \\
& =\mathbb{E}\left[\exp \left(-s \sum_{y_{i} \in \Phi_{d} \backslash\left\{y_{0}\right\}} P_{d} h_{i}\left\|y_{i}\right\|^{-\alpha}\right)\right] \\
& \quad \times \mathbb{E}\left[\exp \left(-s \sum_{x_{i} \in \Phi_{c}} P_{c} g_{i}\left\|x_{i}\right\|^{-\alpha}\right)\right] \\
& =\exp \left(-\lambda_{d} \pi\left(s P_{d}\right)^{\delta} \Gamma(1-\delta) \Gamma(1+\delta)\right) \\
& \quad \times \exp \left(-\lambda_{c} \pi\left(s P_{c}\right)^{\delta} \Gamma(1-\delta) \Gamma(1+\delta)\right) .
\end{aligned}
$$

Therefore,

$$
\mathcal{L}_{I_{d}}\left(P_{d}^{-1} T l^{\alpha}\right)=\exp \left(-\pi\left[\lambda_{d}+\lambda_{c}\left(\frac{P_{c}}{P_{d}}\right)^{\delta}\right] \nu l^{2}\right),
$$

where $\nu$ is given in (8). Then by plugging (1) (20) into (18), we complete the proof.

Remark 1. Via the expressions of $p_{c}$ and $p_{d}$ shown in Theorem 1 and 2, we can observe that $\mu, \nu$ represent the effect of the interference from the cellular links and D2D links respectively, and $\left(P_{d} / P_{c}\right)^{\delta},\left(P_{c} / P_{d}\right)^{\delta}$ can be regarded as the conversion factors of powers.

\section{Stochastic Equivalence of Interference}

By (4) (15), the cumulative interference at each link is generated by two-tier interferers, i.e., the cellular-tier and D2D-tier interferers. The analysis of such two-tier interference is trivial, as shown in the derivations of Theorem 1 and 2. Therefore, to simplify the analysis and facilitate the performance evaluation of SIC in later sections, we propose an approach to equate the two-tier interference by a single-tier interference that has the same stochastic characteristics (in terms of successful transmission probability) as the two-tier interference.

We first study the stochastic equivalence of the interference for cellular links. By (4), the interferers for the typical cellular link constitute $\Phi_{c-\text { intf }}=\left(\Phi_{c} \backslash\left\{x_{0}\right\}\right) \bigcup \Phi_{d}$. We represent $\Phi_{c-i n t f}$ by an equivalent PPP $\Phi_{c-i n t f}^{\mathrm{eq}} \backslash\left\{x_{0}\right\}$ with density $\lambda_{c-i n t f}^{\mathrm{eq}}$ and transmission power $P_{c}$. Then, the equivalent interference at the typical cellular link can be expressed as

$$
I_{c}^{\mathrm{eq}}=\sum_{x_{i} \in \Phi_{c-i n t f}^{\mathrm{eq}} \backslash\left\{x_{0}\right\}} P_{c} g_{i}\left\|x_{i}\right\|^{-\alpha},
$$

which has the same stochastic characteristics as $I_{c}$.

Lemma 1. The density of the equivalent interferers for cellular links is

$$
\lambda_{c-i n t f}^{e q}=\lambda_{c}+\lambda_{d}\left(\frac{P_{d}}{P_{c}}\right)^{\delta} \frac{\nu}{\mu},
$$

where $\mu, \nu$ are given in $(7),(8)$ respectively.

Proof: Considering $I_{c}^{\mathrm{eq}}$ has the same stochastic characteristics as $I_{c}$, we have

$$
\mathcal{L}_{I_{c}}\left(P_{c}^{-1} \operatorname{Tr}^{\alpha}\right)=\mathcal{L}_{I_{c}^{\mathrm{eq}}}\left(P_{c}^{-1} \operatorname{Tr}^{\alpha}\right) .
$$

The Laplace transform of $I_{c}^{\mathrm{eq}}$ is obtained as

$$
\begin{aligned}
& \mathcal{L}_{I_{c}^{\mathrm{eq}}}(s)=\mathbb{E}_{I_{c}^{\mathrm{eq}}}\left[\exp \left(-s I_{c}^{\mathrm{eq}}\right)\right] \\
& =\mathbb{E}\left[\exp \left(-s \sum_{x_{i} \in \Phi_{c-i n t f}^{\mathrm{eq}} \backslash\left\{x_{0}\right\}} P_{c} g_{i}\left\|x_{i}\right\|^{-\alpha}\right)\right] \\
& =\exp \left(-\lambda_{c-i n t f}^{\mathrm{eq}} \pi \frac{\delta}{1-\delta} s P_{c} r^{2-\alpha}{ }_{2} F_{1}\left(1,1-\delta ; 2-\delta ;-\frac{s P_{c}}{r^{\alpha}}\right)\right) .
\end{aligned}
$$

Therefore,

$$
\mathcal{L}_{I_{c}^{\mathrm{eq}}}\left(P_{c}^{-1} \operatorname{Tr}^{\alpha}\right)=\exp \left(-\lambda_{c-\text { int } f}^{\mathrm{eq}} \pi \mu r^{2}\right),
$$

where $\mu$ is given in (7). Then by plugging (13) (25) into (23), we complete the proof.

It is noted that $p_{c}$ can be obtained as $\int_{0}^{\infty} \mathcal{L}_{I_{c}^{\text {eq }}}\left(P_{c}^{-1} T r^{\alpha}\right)$. $f_{r}(r) \mathrm{d} r$, and by (24), we have

$$
p_{c}=\frac{\lambda_{c}}{\lambda_{c-i n t f}^{\mathrm{eq}} \mu+\lambda_{c}},
$$

which is consistent with the result of Theorem 1.

We next study the stochastic equivalence of the interference for D2D links. By (15), the interferers for the typical D2D link constitute $\Phi_{d-\text { intf }}=\left(\Phi_{d} \backslash\left\{y_{0}\right\}\right) \bigcup \Phi_{c}$. We represent 
$\Phi_{d-i n t f}$ by an equivalent PPP $\Phi_{d-i n t f}^{\mathrm{eq}} \backslash\left\{y_{0}\right\}$ with density $\lambda_{d-i n t f}^{\mathrm{eq}}$ and transmission power $P_{d}$. Then, the equivalent interference at the typical D2D link can be expressed as

$$
I_{d}^{\mathrm{eq}}=\sum_{y_{i} \in \Phi_{d-i n t f}^{\mathrm{eq}} \backslash\left\{y_{0}\right\}} P_{d} h_{i}\left\|y_{i}\right\|^{-\alpha}
$$

Lemma 2. The density of the equivalent interferers for $D 2 D$ links is

$$
\lambda_{d-i n t f}^{e q}=\lambda_{d}+\lambda_{c}\left(\frac{P_{c}}{P_{d}}\right)^{\delta}
$$

Proof: Starting from the Laplace transform of $I_{d}^{\mathrm{eq}}$,

$$
\begin{aligned}
& \mathcal{L}_{I_{d}^{\mathrm{eq}}}(s)=\mathbb{E}_{I_{d}^{\mathrm{eq}}}\left[\exp \left(-s I_{d}^{\mathrm{eq}}\right)\right] \\
& =\mathbb{E}\left[\exp \left(\sum_{y_{i} \in \Phi_{d-i n t f}^{\mathrm{eq}} \backslash\left\{y_{0}\right\}} P_{d} h_{i}\left\|y_{i}\right\|^{-\alpha}\right)\right] \\
& =\exp \left(-\lambda_{d-i n t f}^{\mathrm{eq}} \pi\left(s P_{d}\right)^{\delta} \Gamma(1-\delta) \Gamma(1+\delta)\right) .
\end{aligned}
$$

Therefore,

$$
\mathcal{L}_{I_{d}^{\mathrm{eq}}}\left(P_{d}^{-1} T l^{\alpha}\right)=\exp \left(-\lambda_{d-i n t f}^{\mathrm{eq}} \pi \nu l^{2}\right),
$$

where $\nu$ is given in (8). Then by letting $\mathcal{L}_{I_{d}^{\text {eq }}}\left(P_{d}^{-1} T l^{\alpha}\right)=$ $\mathcal{L}_{I_{d}}\left(P_{d}^{-1} T l^{\alpha}\right)$, we complete the proof.

It is noted that $p_{d}$ can be obtained as $\int_{0}^{\infty} \mathcal{L}_{I_{d}^{\mathrm{eq}}}\left(P_{d}^{-1} T l^{\alpha}\right)$. $f_{l}(l) \mathrm{d} l$, and by (30), we have

$$
p_{d}=\frac{\lambda_{d}}{\lambda_{d-i n t f}^{\mathrm{eq}} \nu+\lambda_{d}},
$$

which is consistent with the result of Theorem 2 .

\section{Network Performance With SiC}

In this section, we study how SIC affects the successful transmission probabilities in D2D-enabled cellular networks. The analysis is based on the stochastic equivalence models proposed in section IV.

\section{A. Successful Transmission Probability of Cellular Links}

We conduct the analysis on a typical cellular link that comprises a typical SIC-capable BS located at the origin and its associated cellular user located at $x_{0}$, where $\left\|x_{0}\right\|=r$. The equivalent interferers for the typical cellular link are ordered by their received power at the typical BS such that $P_{c} g_{i}\left\|x_{i}\right\|^{-\alpha}>P_{c} g_{j}\left\|x_{j}\right\|^{-\alpha}, \forall 0<i<j$. Before deriving the successful transmission probability of the typical cellular link, we first present two lemmas.

Given that $n$ strongest equivalent interferers have been canceled, the received SIR of the typical cellular link, i.e., the received SIR at the typical BS, can be expressed as

$$
\operatorname{SIR}_{c}^{(n)}=\frac{P_{c} g_{0} r^{-\alpha}}{I_{c}^{\mathrm{eq}(n)}},
$$

where

$$
I_{c}^{\mathrm{eq}(n)}=\sum_{x_{i} \in \Phi_{c-i n t f}^{\mathrm{eq}} \backslash\left\{x_{0}, x_{1}, \ldots x_{n}\right\}} P_{c} g_{i}\left\|x_{i}\right\|^{-\alpha}
$$

is the cumulative interference for the typical cellular link. Then, the successful transmission probability of the typical cellular link given that $n$ strongest equivalent interferers have been canceled can be defined as

$$
p_{c}^{(n)} \triangleq \mathbb{P}\left[\operatorname{SIR}_{c}^{(n)}>T\right] \text {. }
$$

The expression of $p_{c}^{(n)}$ is given by the following lemma.

Lemma 3. Given that $n$ strongest equivalent interferers have been canceled, the successful transmission probability of the typical cellular link is

$$
\begin{aligned}
& p_{c}^{(n)}= \\
& \int_{0}^{\infty} \int_{0}^{\infty} \frac{2\left(\lambda_{c-i n t f}^{e q} \pi d_{n}^{2}\right)^{n}}{d_{n} \Gamma(n)} e^{-\lambda_{c-i n t f}^{e q} \pi \xi\left(r, d_{n}\right)} f_{r}(r) \mathrm{d} d_{n} \mathrm{~d} r,
\end{aligned}
$$

where $f_{r}(r)$ is given in (2) and

$\xi\left(r, d_{n}\right)=\frac{\delta}{1-\delta} \operatorname{Tr}^{\alpha} d_{n}^{2-\alpha}{ }_{2} F_{1}\left(1,1-\delta ; 2-\delta ;-\frac{T r^{\alpha}}{d_{n}^{\alpha}}\right)+d_{n}^{2}$.

Proof: Starting from the definitions of $p_{c}^{(n)}$ and $\operatorname{SIR}_{c}^{(n)}$,

$$
\begin{aligned}
p_{c}^{(n)} & \triangleq \mathbb{P}\left[\operatorname{SIR}_{c}^{(n)}>T\right]=\mathbb{E}_{r}\left[\mathcal{L}_{I_{c}^{\mathrm{eq}(n)}}\left(P_{c}^{-1} T r^{\alpha}\right)\right] \\
& =\int_{0}^{\infty} \mathcal{L}_{I_{c}^{\mathrm{eq}(n)}}\left(P_{c}^{-1} \operatorname{Tr}^{\alpha}\right) \cdot f_{r}(r) \mathrm{d} r .
\end{aligned}
$$

The calculation of $\mathcal{L}_{I_{c}^{\mathrm{eq}(n)}}$ requires the distribution of the sum of the order statistics of interfering powers, which is difficult to obtain in the SIC scenario. However, it has been shown that the order statistics of received powers in modern networks are dominated by the distance [29]. Therefore, we can calculate $\mathcal{L}_{I_{c}^{\mathrm{eq}(n)}}$ by relaxing the ordering of interfering powers to that of interfering distances. Denote the distance from $n$-th equivalent interferer to the origin by $d_{n}$, then we have

$$
\begin{aligned}
& \mathcal{L}_{I_{c}^{\mathrm{eq}(n)}}(s)=\mathbb{E}\left[\exp \left(-s I_{c}^{\mathrm{eq}(n)}\right)\right] \\
& =\mathbb{E}_{d_{n}, \Phi_{c-i n t f}^{\mathrm{eq}}, g}\left[\prod_{x_{i} \in \Phi_{c-i n t f}^{\mathrm{eq}} \backslash\left\{x_{0}, x_{1}, \ldots x_{n}\right\}} e^{-s P_{c} g_{i}\left\|x_{i}\right\|^{-\alpha}}\right] \\
& =\mathbb{E}_{d_{n}}\left[e^{\left.-\lambda_{c-i n t f}^{\mathrm{eq}} \int_{\Phi_{c-i n t f}^{\mathrm{eq}} \cap \bar{B}\left(o, d_{n}\right)}\left(1-\mathbb{E}_{g}\left[e^{-s P_{c} g_{i}\left\|x_{i}\right\|^{-\alpha}}\right]\right) \mathrm{d} x_{i}\right]}\right.
\end{aligned}
$$

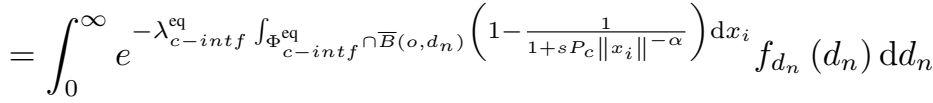

$$
\begin{aligned}
& =\int_{0}^{\infty} e^{-\lambda_{c-i n t f}^{\mathrm{eq}} \cdot 2 \pi \int_{d_{n}}^{\infty} \frac{v}{1+s^{-1} P_{c}^{-1} v^{\alpha}} \mathrm{d} v} \cdot f_{d_{n}}\left(d_{n}\right) \mathrm{d} d_{n} \\
& =\int_{0}^{\infty} e^{-\lambda_{c-i n t f}^{\mathrm{eq}} \pi \frac{\delta}{1-\delta} s P_{c} d_{n}^{2-\alpha}{ }_{2} F_{1}\left(1,1-\delta ; 2-\delta ;-\frac{s P_{c}}{d_{n}^{\alpha}}\right)} f_{d_{n}}\left(d_{n}\right) \mathrm{d} d_{n} .
\end{aligned}
$$

From [30], the probability density function of $d_{n}$ is given by

$$
f_{d_{n}}\left(d_{n}\right)=e^{-\lambda_{c-i n t f}^{\mathrm{eq}} \pi d_{n}^{2}} \cdot \frac{2\left(\lambda_{c-i n t f}^{\mathrm{eq}} \pi d_{n}^{2}\right)^{n}}{d_{n} \Gamma(n)} .
$$


By plugging (39) into (38) and letting $s=P_{c}^{-1} T r^{\alpha}$, we get

$$
\begin{aligned}
& \mathcal{L}_{I_{c}^{\mathrm{eq}(n)}}\left(P_{c}^{-1} T r^{\alpha}\right) \\
& =\int_{0}^{\infty} e^{-\lambda_{c-i n t f}^{\mathrm{eq}} \pi \xi\left(r, d_{n}\right)} \cdot \frac{2\left(\lambda_{c-i n t f}^{\mathrm{eq}} \pi d_{n}^{2}\right)^{n}}{d_{n} \Gamma(n)} \mathrm{d} d_{n},
\end{aligned}
$$

where $\xi\left(r, d_{n}\right)=\frac{\delta}{1-\delta} T r^{\alpha} d_{n}^{2-\alpha}{ }_{2} F_{1}\left(1,1-\delta ; 2-\delta ;-\frac{T r^{\alpha}}{d_{n}^{\alpha}}\right)+$ $d_{n}^{2}$. Then by plugging (2) (40) into (37), we complete the proof.

A possible approach for simplifying the expression of $p_{c}^{(n)}$ is to approximate $d_{n}$ by a fixed value $\tilde{d}_{n}$, which equals the expectation of $d_{n}$, i.e.,

$$
\tilde{d}_{n}=\mathbb{E}\left[d_{n}\right]=\frac{\Gamma\left(n+\frac{1}{2}\right)}{\sqrt{\pi \lambda_{c-i n t f}^{\mathrm{eq}} \Gamma(n)} .}
$$

Then, $p_{c}^{(n)}$ can be approximated by

$$
p_{c}^{(n)}=\int_{0}^{\infty} e^{-\lambda_{c-i n t f}^{\mathrm{eq}} \pi \tilde{\xi}\left(r, \tilde{d_{n}}\right)} \cdot f_{r}(r) \mathrm{d} r,
$$

where $f_{r}(r)$ is given in $(2)$ and

$$
\tilde{\xi}\left(r, \tilde{d_{n}}\right)=\frac{\delta}{1-\delta} \operatorname{Tr}^{\alpha}{\tilde{d_{n}}}^{2-\alpha}{ }_{2} F_{1}\left(1,1-\delta ; 2-\delta ;-\frac{T r^{\alpha}}{{\tilde{d_{n}}}^{\alpha}}\right) \text {. }
$$

Next, we study the probability of canceling $n$-th strongest equivalent interferer. Given that all $n-1$ stronger equivalent interferers have been canceled, the received SIR of $n$-th strongest interferer at the typical BS can be expressed as

$$
\mathrm{SIR}_{c-i n t f}^{(n)}=\frac{P_{c} g_{n}\left\|x_{n}\right\|^{-\alpha}}{I_{c-i n t f}^{\mathrm{eq}(n)}},
$$

where

$$
I_{c-i n t f}^{\mathrm{eq}(n)}=\sum_{x_{i} \in \Phi_{c-i n t f}^{\mathrm{eq}} \backslash\left\{x_{n}, x_{1}, \ldots x_{n-1}\right\}} P_{c} g_{i}\left\|x_{i}\right\|^{-\alpha}
$$

is the cumulative interference for $n$-th strongest equivalent interferer. Then, the probability of canceling (decoding) $n$ th strongest equivalent interferer given that all $n-1$ stronger equivalent interferers have been canceled can be defined as

$$
p_{c-i n t f}^{(n)} \triangleq \mathbb{P}\left[\operatorname{SIR}_{c-i n t f}^{(n)}>T\right] .
$$

The expression of $p_{c-i n t f}^{(n)}$ is given by the following lemma.

Lemma 4. Given that all $n-1$ stronger equivalent interferers have been canceled, the probability of canceling $n$-th strongest equivalent interferer for the typical cellular link is

$$
p_{c-i n t f}^{(n)}=\frac{1}{(\mu+1)^{n}},
$$

where $\mu$ is given in (7).

Proof: Following the relaxation approach for the order statistics of interfering powers in the proof of Lemma 3, we can rewrite $\operatorname{SIR}_{c-i n t f}^{(n)}$ as

$$
\mathrm{SIR}_{c-i n t f}^{(n)}=\frac{P_{c} g_{n} d_{n}^{-\alpha}}{I_{c-i n t f}^{\mathrm{eq}(n)}} .
$$

Then, we have

$$
\begin{aligned}
& p_{c-i n t f}^{(n)} \triangleq \mathbb{P}\left[\operatorname{SIR}_{c-i n t f}^{(n)}>T\right] \\
& =\mathbb{E}_{d_{n}, I_{c-i n t f}^{\mathrm{eq}(n)}}\left[\exp \left(-P_{c}^{-1} T d_{n}^{\alpha} I_{c-i n t f}^{\mathrm{eq}(n)}\right)\right] \\
& =\mathbb{E}_{d_{n}}\left[\mathcal{L}_{I_{c-i n t f}^{\mathrm{eq}(n)}}\left(P_{c}^{-1} T d_{n}^{\alpha}\right)\right] \\
& =\int_{0}^{\infty} \mathcal{L}_{I_{c-i n t f}^{\mathrm{eq}(n)}}\left(P_{c}^{-1} T d_{n}^{\alpha}\right) \cdot f_{d_{n}}\left(d_{n}\right) \mathrm{d} d_{n} .
\end{aligned}
$$

The Laplace transform of $I_{c-i n t f}^{\mathrm{eq}(n)}$ is obtained as

$$
\mathcal{L}_{I_{c-i n t f}^{\mathrm{eq}(n)}}(s)=e^{-\lambda_{c-i n t f}^{\mathrm{eq}} \pi \frac{\delta}{1-\delta} s P_{c} d_{n}^{2-\alpha} F_{2} F_{1}\left(1,1-\delta ; 2-\delta ;-\frac{s P_{c}}{d_{n}^{\alpha}}\right)} .
$$

Hence,

$$
\mathcal{L}_{I_{c-i n t f}^{\mathrm{eq}(n)}}\left(P_{c}^{-1} T d_{n}^{\alpha}\right)=\exp \left(-\lambda_{c-i n t f}^{\mathrm{eq}} \pi \mu d_{n}^{2}\right),
$$

where $\mu$ is given in (7). Then by plugging (39) (51) into (49), we complete the proof.

Based on Lemma 3 and 4, we can derive the successful transmission probability of the typical cellular link.

Theorem 3. The successful transmission probability of cellular links with infinite SIC capability is

$$
p_{c}^{S I C}=p_{c}+\sum_{n=1}^{\infty}\left(\prod_{i=1}^{n} p_{c-i n t f}^{(i)}\right)\left(\prod_{i=0}^{n-1}\left(1-p_{c}^{(i)}\right)\right) p_{c}^{(n)},
$$

where $p_{c}, p_{c}^{(n)}, p_{c-\text { int } f}^{(i)}$ are given in $(6),(35),(47)$ respectively.

Proof: For the typical cellular link, we define the event of its successful transmission without SIC as

$$
E_{0}: \operatorname{SIR}_{c}^{(0)}>T,
$$

and the event of its successful transmission with $n$-level $(n \geq 1)$ SIC as

$E_{n}:\left(\bigcap_{i=1}^{n} \operatorname{SIR}_{c-i n t f}^{(i)}>T\right) \cap\left(\bigcap_{i=0}^{n-1} \operatorname{SIR}_{c}^{(i)}<T\right) \cap\left(\operatorname{SIR}_{c}^{(n)}>T\right)$.

Using the assumption that the interference to each user is independent, we get

$\mathbb{P}\left[E_{n}\right]= \begin{cases}p_{c}^{(0)}=p_{c}, & n=0, \\ \left(\prod_{i=1}^{n} p_{c-i n t f}^{(i)}\right)\left(\prod_{i=0}^{n-1}\left(1-p_{c}^{(i)}\right)\right) p_{c}^{(n)}, & n \geq 1 .\end{cases}$

Therefore, the successful transmission probability of cellular links can be obtained as $p_{c}^{\mathrm{SIC}}=\sum_{n=0}^{\infty} \mathbb{P}\left[E_{n}\right]$.

Corollary 1. The successful transmission probability of cellular links with (finite) $N$-level $(N \geq 1)$ SIC capability is

$$
p_{c}^{N-S I C}=p_{c}+\sum_{n=1}^{N}\left(\prod_{i=1}^{n} p_{c-i n t f}^{(i)}\right)\left(\prod_{i=0}^{n-1}\left(1-p_{c}^{(i)}\right)\right) p_{c}^{(n)},
$$

where $p_{c}, p_{c}^{(n)}, p_{c-i n t f}^{(i)}$ are given in $(6),(35),(47)$ respectively. 


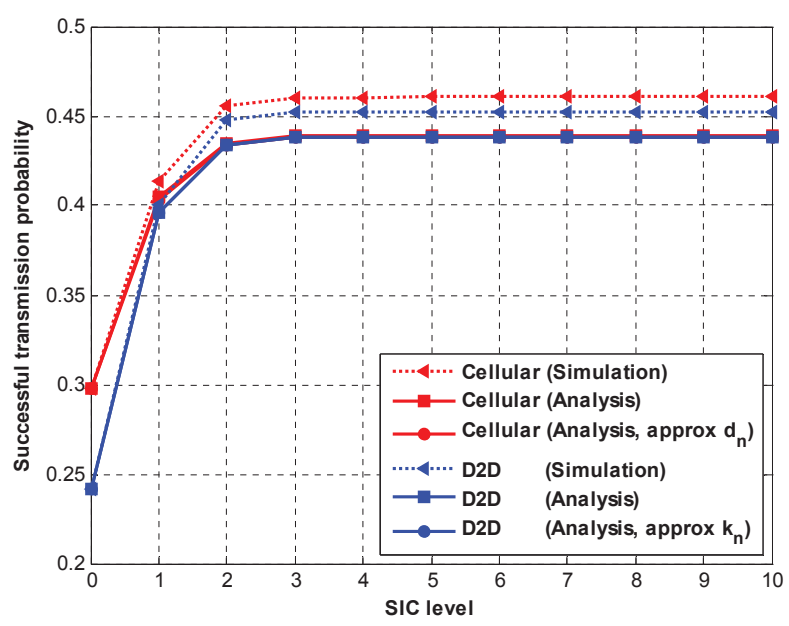

Figure 3: Successful transmission probabilities of cellular and D2D links with SIC.

\section{B. Successful Transmission Probability of D2D Links}

We consider a typical D2D link with $M$-level SIC receivers. The assumption of finite-level SIC receiver is motivated by the limited computational capabilities of D2D users. The derivation of the successful transmission probability of D2D links is quite similar to that of cellular links, and hence we directly present the results and omit their proofs.

Lemma 5. Given that $n$ strongest equivalent interferers have been canceled, the successful transmission probability of the typical D2D link is

$$
\begin{aligned}
& p_{d}^{(n)}= \\
& \int_{0}^{\infty} \int_{0}^{\infty} \frac{2\left(\lambda_{d-i n t f}^{e q} \pi k_{n}^{2}\right)^{n}}{k_{n} \Gamma(n)} e^{-\lambda_{d-i n t f}^{e q} \pi \kappa\left(l, k_{n}\right)} f_{l}(l) \mathrm{d} k_{n} \mathrm{~d} l,
\end{aligned}
$$

where $f_{l}(l)$ is given in (1) and

$\kappa\left(l, k_{n}\right)=\frac{\delta}{1-\delta} T l^{\alpha} k_{n}^{2-\alpha}{ }_{2} F_{1}\left(1,1-\delta ; 2-\delta ;-\frac{T l^{\alpha}}{k_{n}^{\alpha}}\right)+k_{n}^{2}$.

Lemma 6. Given that all $n-1$ stronger equivalent interferers have been canceled, the probability of canceling $n$-th strongest equivalent interferer for the typical D2D link is

$$
p_{d-i n t f}^{(n)}=\frac{1}{(\mu+1)^{n}},
$$

where $\mu$ is given in (7).

Theorem 4. The successful transmission probability of $D 2 D$ links with (finite) $M$-level $(M \geq 1)$ SIC capability is

$$
p_{d}^{M-S I C}=p_{d}+\sum_{n=1}^{M}\left(\prod_{i=1}^{n} p_{d-i n t f}^{(i)}\right)\left(\prod_{i=0}^{n-1}\left(1-p_{d}^{(i)}\right)\right) p_{d}^{(n)},
$$

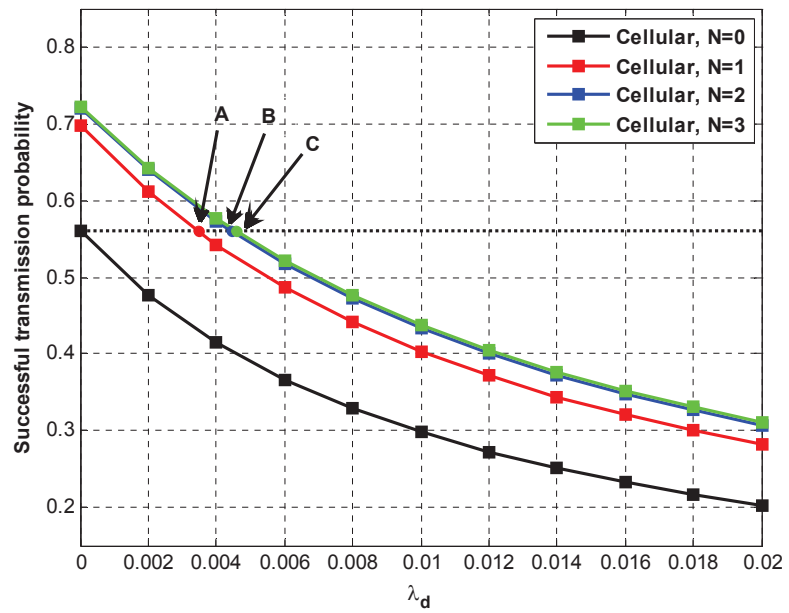

Figure 4: Successful transmission probability of cellular links with SIC vs. density of D2D links.

where $p_{d}, p_{d}^{(n)}, p_{d-\text { intf }}^{(n)}$ are given in $(17),(57),(59)$ respectively.

\section{Discussions and Numerical Results}

Now that we have developed expressions for the successful transmission probabilities of cellular and D2D links with SIC capabilities, based on the stochastic equivalence models. It is noted that the derived analytical expressions are not the exact results of corresponding successful transmission probabilities, since approximated models are used in the derivation. We provide some numerical results to compare the analytical results with the actual (simulation) results. The system parameters are set as $\alpha=4, P_{c}=P_{d}=1, \lambda_{c}=\lambda_{d}=0.01, T=1$.

Fig. 3 shows the successful transmission probabilities with SIC capabilities. As can be observed, there exist gaps between the analytical results of $p_{c}^{N-S I C}, p_{d}^{M-S I C}$ and corresponding simulation results. The analytical results can be regarded as lower bounds on the successful transmission probabilities. The analytical results based on approximated $d_{n}$ (see (41) (42)) and $k_{n}$ are also plotted in the figure, through which we can find that the results of the approximated analytical expressions closely match those of the exact analytical expressions. Therefore, the approximated analytical expressions can be employed to simplify the calculation of the successful transmission probabilities. In addition, as shown in the figure, 2-level SIC can provide almost $50 \%$ performance improvement for the network; however, when the SIC level is larger than 2, SIC cannot further improve the network performance. Considering the hardware cost of multi-level SIC, 1- and 2-level SIC can be adopted in practical networks.

Fig.4 shows the successful transmission probability of cellular links with SIC vs. the density of D2D links. As expected, increasing the density of D2D links leads to a decrease in the successful transmission probability of cellular links. However, from this figure we can observe that SIC can compensate part of the performance loss of cellular links. For example, 1-level 
SIC at the cellular receiver can compensate the performance loss generated by D2D interferers of density $\lambda_{d}=0.0035$ (see point A), and 2-level SIC corresponds to $\lambda_{d}=0.0045$ (see point B).

\section{CONCLUSION}

In this paper, we study the performance of SIC in largescale D2D-enabled cellular networks using the tools from stochastic geometry. We derive the successful transmission probabilities of the network without SIC as the baseline results. To simplify the interference analysis, we propose the approach of stochastic equivalence of the interference, by which the twotier interference can be represented by an equivalent single-tier interference. Based on the stochastic equivalence models, we derive the successful transmission probabilities of cellular and D2D links with infinite and finite SIC capabilities. The SIC gains are validated by analytical and numerical results.

\section{ACKNOWLEDGMENT}

This work is supported by NSF China (No.61325012, 61271219, 61221001, 61202373, 61102052, 61102051, 61428205); China Ministry of Education Doctor Program (No.20130073110025); National Mobile Communications Research Laboratory, Southeast University (No.2012D13, 2014D07); Shanghai Basic Research Key Project (12JC1405200, 11JC1405100); Shanghai International Cooperation Project (No.13510711300); National Basic Research 973 Program of China (No.2015CB352403); Jiangsu Future Network Research Project (BY2013095-1-10).

\section{REFERENCES}

[1] K. Doppler, M. Rinne, C. Wijting, C. Ribeiro, and K. Hugl, "Device-todevice communication as an underlay to LTE-advanced networks," IEEE Communications Magazine, vol. 47, no. 12, pp. 42-49, Dec. 2009.

[2] G. Fodor, E. Dahlman, G. Mildh, S. Parkvall, N. Reider, G. Miklos, and Z. Turanyi, "Design aspects of network assisted device-to-device communications," IEEE Communications Magazine, vol. 50, no. 3, pp. 170-177, Mar. 2012.

[3] Wireless World Initiative New Radio - WINNER+ D2.1, "Preliminary WINNER+ system concept," 2009.

[4] 3GPP TS 23.303, "Technical specification group services and system aspects; Proximity-based services (ProSe)," Rel-12, 2014.

[5] C. Xu, L. Song, Z. Han, Q. Zhao, X. Wang, X. Cheng, and B. Jiao, "Efficiency resource allocation for device-to-device underlay communication systems: A reverse iterative combinatorial auction based approach," IEEE Journal on Selected Areas in Communications, vol. 31, no. 9, pp. 348-358, Sep. 2013.

[6] B. Kaufman, J. Lilleberg, and B. Aazhang, "Spectrum sharing scheme between cellular users and ad-hoc device-to-device users," IEEE Transactions on Wireless Communications, vol. 12, no. 3, pp. 1038-1049, Mar. 2013.

[7] Y. Pei and Y.-C. Liang, "Resource allocation for device-to-device communications overlaying two-way cellular networks," IEEE Transactions on Wireless Communications, vol. 12, no. 7, pp. 3611-3621, July 2013.

[8] H. Min, W. Seo, J. Lee, S. Park, and D. Hong, "Reliability improvement using receive mode selection in the device-to-device uplink period underlaying cellular networks," IEEE Transactions on Wireless Communications, vol. 10, no. 2, pp. 413-418, Feb. 2011.

[9] C. Ma, G. Sun, X. Tian, K. Ying, H. Yu, and X. Wang, "Cooperative relaying schemes for device-to-device communication underlaying cellular networks," in Proceedings of 2013 IEEE Global Communications Conference (GLOBECOM), Dec. 2013, pp. 3890-3895.

[10] G. Boudreau, J. Panicker, N. Guo, R. Chang, N. Wang, and S. Vrzic, "Interference coordination and cancellation for $4 \mathrm{G}$ networks," IEEE Communications Magazine, vol. 47, no. 4, pp. 74-81, Apr. 2009.
[11] J. Andrews, "Interference cancellation for cellular systems: a contemporary overview," IEEE Wireless Communications, vol. 12, no. 2, pp. 19-29, Apr. 2005

[12] E. Gelal, K. Pelechrinis, T.-S. Kim, I. Broustis, S. Krishnamurthy, and B. Rao, "Topology control for effective interference cancellation in multi-user MIMO networks," in Proceedings of 2010 IEEE INFOCOM, Mar. 2010, pp. 1-9

[13] C. Jiang, Y. Shi, Y. Hou, W. Lou, S. Kompella, and S. Midkiff, "Squeezing the most out of interference: An optimization framework for joint interference exploitation and avoidance," in Proceedings of 2012 IEEE INFOCOM, Mar. 2012, pp. 424-432.

[14] C. Xu, L. Ping, P. Wang, S. Chan, and X. Lin, "Decentralized power control for random access with successive interference cancellation," IEEE Journal on Selected Areas in Communications, vol. 31, no. 11, pp. 2387-2396, Nov. 2013.

[15] S. Lv, W. Zhuang, M. Xu, X. Wang, C. Liu, and X. Zhou, "Understanding the scheduling performance in wireless networks with successive interference cancellation," IEEE Transactions on Mobile Computing, vol. 12 , no. 8, pp. 1625-1639, Aug. 2013.

[16] M. Mollanoori and M. Ghaderi, "Uplink scheduling in wireless networks with successive interference cancellation," IEEE Transactions on Mobile Computing, vol. 13, no. 5, pp. 1132-1144, May 2014.

[17] M. Haenggi, J. Andrews, F. Baccelli, O. Dousse, and M. Franceschetti, "Stochastic geometry and random graphs for the analysis and design of wireless networks," IEEE Journal on Selected Areas in Communications, vol. 27, no. 7, pp. 1029-1046, Sep. 2009.

[18] R. Vaze and R. Heath, "Transmission capacity of ad-hoc networks with multiple antennas using transmit stream adaptation and interference cancellation," IEEE Transactions on Information Theory, vol. 58, no. 2, pp. 780-792, Feb. 2012.

[19] M. Haenggi, “The local delay in poisson networks," IEEE Transactions on Information Theory, vol. 59, no. 3, pp. 1788-1802, Mar. 2013.

[20] J. Andrews, F. Baccelli, and R. Ganti, "A tractable approach to coverage and rate in cellular networks," IEEE Transactions on Communications, vol. 59, no. 11, pp. 3122-3134, Nov. 2011.

[21] T. Novlan, H. Dhillon, and J. Andrews, "Analytical modeling of uplink cellular networks," IEEE Transactions on Wireless Communications, vol. 12, no. 6, pp. 2669-2679, Jun. 2013.

[22] X. Lin and J. G. Andrews, "Optimal spectrum partition and mode selection in device-to-device overlaid cellular networks," in Proceedings of 2013 IEEE Global Communications Conference (GLOBECOM), Dec. 2013, pp. 1837-1842.

[23] X. Lin, R. Ratasuk, A. Ghosh, and J. Andrews, "Modeling, analysis and optimization of multicast device-to-device transmissions," IEEE Transactions on Wireless Communications, vol. PP, no. 99, 2014.

[24] Q. Ye, M. Al-Shalash, C. Caramanis, and J. G. Andrews, "Deviceto-device modeling and analysis with a modified matern hardcore BS location model," in Proceedings of 2013 IEEE Global Communications Conference (GLOBECOM), Dec. 2013, pp. 1825-1830.

[25] S. P. Weber, J. G. Andrews, X. Yang, and G. De Veciana, "Transmission capacity of wireless ad hoc networks with successive interference cancellation," IEEE Transactions on Information Theory, vol. 53, no. 8, pp. 2799-2814, Aug. 2007.

[26] J. Lee, J. Andrews, and D. Hong, "Spectrum-sharing transmission capacity with interference cancellation," IEEE Transactions on Communications, vol. 61, no. 1, pp. 76-86, Jan. 2013.

[27] J. Blomer and N. Jindal, "Transmission capacity of wireless ad hoc networks: Successive interference cancellation vs. joint detection," in Proceedings of 2009 IEEE International Conference on Communications (ICC), Jun. 2009, pp. 1-5.

[28] X. Zhang and M. Haenggi, "The aggregate throughput in random wireless networks with successive interference cancellation," in Proceedings of 2013 IEEE International Symposium on Information Theory Proceedings (ISIT), July 2013, pp. 251-255.

[29] M. Wildemeersch, T. Q. Quek, M. Kountouris, and C. H. Slump, "Successive interference cancellation in uplink cellular networks," in Proceedings of 2013 IEEE Workshop on Signal Processing Advances in Wireless Communications (SPAWC), Jun. 2013, pp. 310-314.

[30] M. Haenggi, Stochastic geometry for wireless networks. Cambridge University Press, 2012.

[31] I. S. Gradshteyn, I. M. Ryzhik, A. Jeffrey, and D. Zwillinger, Table of integrals, series, and products. Elsevier/Academic Press, 2007.

[32] F. Baccelli and B. Blaszczyszyn, Stochastic Geometry and Wireless Networks. Now Publishers Inc, 2009. 